\title{
SIGN PATTERNS OF THE SCHWARZ MATRICES AND GENERALIZED HURWITZ POLYNOMIALS*
}

\author{
MIKHAIL TYAGLOV ${ }^{\dagger}$
}

\begin{abstract}
The direct and inverse spectral problems are solved for a wide subclass of the class of Schwarz matrices. A connection between Schwarz matrices and the so-called generalized Hurwitz polynomials is found. The known results due to H. Wall and O. Holtz are briefly reviewed and obtained as particular cases.
\end{abstract}

Key words. Schwarz matrices, inverse spectral problem, generalized Hurwitz polynomials, continued fractions, tridiagonal matrices.

AMS subject classifications. 15A29, 47A75, 47B36, 26C10.

1. Introduction. In this work, we consider the matrices of the form

$$
\left[\begin{array}{cccccc}
-b_{0} & 1 & 0 & \ldots & 0 & 0 \\
-b_{1} & 0 & 1 & \ldots & 0 & 0 \\
0 & -b_{2} & 0 & \ldots & 0 & 0 \\
\vdots & \vdots & \vdots & \ddots & \vdots & \vdots \\
0 & 0 & 0 & \ldots & 0 & 1 \\
0 & 0 & 0 & \ldots & -b_{n-1} & 0
\end{array}\right], \quad b_{k} \in \mathbb{R} \backslash\{0\}
$$

which are usually called the Schwarz matrices. Note that in [16] Schwarz also considered matrices whose $(1,1)$-entry is zero while $(n, n)$-entry is not. Sometimes such matrices are called the Schwarz matrices as well, see $[\underline{8}$. In this paper, we solve direct and inverse problems for such matrices with certain sign patterns.

At first, let us note that such matrices (more exactly, their characteristic polynomials for the case of $\left.b_{k}>0, k=0, \ldots, n-1\right)$ were considered first time by Wall in his study [18] on Hurwitz stable polynomials, i.e. the polynomials with zeroes in the open left half-plane. Schwarz [16] extended Wall's result to real nonzero $b_{k}$, and applied

*Received by the editors on January 3, 2012. Accepted for publication on November 3, 2012 Handling Editor: Carlos Fonseca.

†Shanghai Jiao Tong University, Department of Mathematics, 800 Dong Chuan Road, 200240, Shanghai, China (tyaglov@sjtu.edu.cn). The work was performed at Technische Universität Berlin and at Shanghai Jiao Tong University and was supported by the European Research Council under the European Union's Seventh Framework Programme (FP7/2007-2013)/ERC grant agreement no. 259173 . 
purely matrix methods to study matrices similar to the matrices of the form (1.1). Thus these matrices should be called rather Wall-Schwarz matrices.

The matrices (1.1) are well-studied from the matrix theory point of view (see e.g. [3, 15, 4, 5, 6, 7] and references there). Here we use the method due to Wall [18, 19] to solve the inverse spectral problem, and use our results on the generalized Hurwitz polynomials to solve the direct spectral problem for the Schwarz matrices (1.1) with certain sign patterns.

The case of all $b_{k}$ positive was considered by Wall [18, and later by Schwarz [16] and many other authors. The case of all $b_{k}$ negative was studied by Holtz [13]. Moreover, the case $b_{1}>0, b_{2}, \ldots, b_{n-1}<0$ was considered in [1]. The present work deals with certain intermediate cases between positive and negative $b_{k}$, that is, we study a number of sign patterns for $b_{k}$. Our results include, of course, the results by Wall [18] and by Holtz [13. Such a generalization becomes possible due to relationships between the entries $b_{k}$ of the matrix (1.1) and the Hurwitz determinants (see (2.5)) of its characteristic polynomial.Those relationships were found by Wall in [18. The Hurwitz stable polynomials studied by Wall and the polynomials appeared in the paper by Holtz [13. turned out to be connected by a one-to-one correspondence [17. (see Theorem 4.7 of the present paper).

In [17] it was also discovered that there exists a general class of polynomials whose distribution of zeroes can be described by signs of their Hurwitz determinants, which includes Hurwitz stable polynomials as a subclass, the so-called generalized Hurwitz polynomials. So the Wall's relationship mentioned above gives a possibility to study all matrices of the form (1.1) whose characteristic polynomials are generalized Hurwitz, and to solve the direct and inverse spectral problems for them. Recall also that Schwarz in his paper [16] combined the same relationship and the Routh-Hurwitz theorem to study the location of the spectra of the matrices (1.1) with respect to the imaginary axis. Thus the present work generalizes results by Wall [18], Holtz [13], and Bebiano and Providência [1], and specifies some results by Schwarz [16].

The paper is organized as follows. In Section 2 we discuss some results by Wall [18] and obtain a consequence that may be new (see Theorem 2.2). In this section we also introduce some constructions and auxiliary results that will be of use in what follows. For the sake of the reader's convenience, Section 3 is devoted to known results on the direct and inverse problems for the matrices of the form (1.1). We present the results by Wall, Schwarz, and Holtz in the form that allows the reader to easily compare their results with results of the next sections and to understand what new was done by the author. In Section 4 we recall some basic facts on the generalized Hurwitz polynomials established in [17. In Section 5 we prove our main theorems on the direct and inverse problems for Schwarz matrices with certain sign patterns. Note that we state our results in the same form as Schwarz did in [16, Satz 5] (see 
Theorem 3.4 of the present work). Finally, in Section 6 we consider an example and show how our results of Section 5 can be applied to matrices (1.1) with one sign change in the sequence $b_{1}, \ldots, b_{n-1}$. In particular, we solve the direct and inverse problems for the matrices (1.1) with $b_{1}>0, b_{2}, \ldots, b_{n-1}<0$ considered in [1. Note, only the direct spectral problem is studied in [1].

2. Wall's continued fractions and the Schwarz matrices. Given a monic real polynomial

$$
p(z)=z^{n}+a_{1} z^{n-1}+\cdots+a_{n}
$$

we represent it as follows

$$
p(z)=p_{0}\left(z^{2}\right)+z p_{1}\left(z^{2}\right),
$$

where the polynomials $p_{0}(u)$ and $p_{1}(u)$ are the even and odd parts of the polynomial $p$, respectively:

$$
\begin{gathered}
p_{0}(u)=a_{n}+a_{n-2} u+a_{n-4} u^{2}+\cdots, \\
p_{1}(u)=a_{n-1}+a_{n-3} u+a_{n-5} u^{2}+\cdots,
\end{gathered}
$$

and introduce the following polynomial

$$
q(z)=\left\{\begin{array}{lll}
p_{0}\left(z^{2}\right) & \text { if } & n=2 l+1 \\
z p_{1}\left(z^{2}\right) & \text { if } & n=2 l
\end{array}\right.
$$

With the polynomial $p$ we associate also determinants called the Hurwitz determinants:

$$
\Delta_{j}(p)=\operatorname{det}\left[\begin{array}{cccccc}
a_{1} & a_{3} & a_{5} & a_{7} & \ldots & a_{2 j-1} \\
1 & a_{2} & a_{4} & a_{6} & \ldots & a_{2 j-2} \\
0 & a_{1} & a_{3} & a_{5} & \ldots & a_{2 j-3} \\
0 & 1 & a_{2} & a_{4} & \ldots & a_{2 j-4} \\
\vdots & \vdots & \vdots & \vdots & \ddots & \vdots \\
0 & 0 & 0 & 0 & \ldots & a_{j}
\end{array}\right], \quad j=1, \ldots, n
$$

where we set $a_{i} \equiv 0$ for $i>n$.

In 1945, H.S. Wall established [18] (see also [19]) the following theorem.

THEOREM 2.1 (Wall). If the coefficients of the polynomial p given in (2.1) satisfy the inequalities

$$
\Delta_{j}(p) \neq 0, \quad j=1, \ldots, n,
$$


then there is a uniquely determined continued fraction of the form

$$
\frac{q(z)}{p(z)}=\frac{b_{0}}{z+b_{0}+\frac{b_{1}}{z+\frac{b_{2}}{\ddots+\frac{b_{n-1}}{z}}}}
$$

where $q$ is defined in (2.4) and the real coefficients $b_{k}$ are given by the formula

$$
b_{0}=\Delta_{1}(p),
$$

$$
b_{k}=\frac{\Delta_{k-2}(p) \Delta_{k+1}(p)}{\Delta_{k-1}(p) \Delta_{k}(p)}, \quad k=1, \ldots, n-1,
$$

where $\Delta_{-1}(p)=\Delta_{0}(p) \equiv 1$.

Conversely, the coefficients in the last denominator of a continued fraction of the form (2.7) satisfy the inequalities (2.6).

From the form of the continued fraction (2.7) it is easy to see that

$$
\frac{q(z)}{p(z)}=b_{0}\left(\left(z E_{n}-J_{n}\right)^{-1} e_{1}, e_{1}\right),
$$

where $e_{1}$ is the first coordinate vector in $\mathbb{R}^{n}, E_{n}$ is the $n \times n$ unity matrix, and

$$
J_{n}=\left[\begin{array}{cccccc}
-b_{0} & 1 & 0 & \ldots & 0 & 0 \\
-b_{1} & 0 & 1 & \ldots & 0 & 0 \\
0 & -b_{2} & 0 & \ldots & 0 & 0 \\
\vdots & \vdots & \vdots & \ddots & \vdots & \vdots \\
0 & 0 & 0 & \ldots & 0 & 1 \\
0 & 0 & 0 & \ldots & -b_{n-1} & 0
\end{array}\right]
$$

where the nonzero real entries $b_{k}$ are exactly the coefficients of the continued fraction (2.7). In other words, the polynomial $p(z)$ is the characteristic polynomial of the matrix $J_{n}$, while the polynomial $q(z) / b_{0}$ is the characteristic polynomial of the principal submatrix of the matrix $J_{n}$ obtained by deleting the first column and the first row. Thus, we come to the following consequence.

THEOREM 2.2. The characteristic polynomial $p$ of the matrix $J_{n}$ defined in (2.9) satisfies the inequalities

$$
\Delta_{j}(p) \neq 0, \quad j=1, \ldots, n .
$$

Conversely, for every real polynomial p satisfying the inequalities (2.6), there exists a unique matrix $J_{n}$ of the form (2.9) whose characteristic polynomial is $p$. 
The matrices of the form (2.9) are called the Schwarz matrices after H. Schwarz who developed a method for transforming a given nonderogatory matrix with the characteristic polynomial, satisfying the inequalities (2.6), to the form (2.9) (see [16]). We again pay attention of the reader to the fact that Schwarz considered $(n, n)$-entry nonzero rather than $(1,1)$-entry nonzero as we do. In this work, we follow H. Wall who considered tridiagonal matrices of the form (2.9) earlier than Schwarz.

Theorem 2.2 provides a solution of somewhat direct and inverse problems for tridiagonal matrices of the form (2.9). These problems, however, are not spectral and concern properties of the characteristic polynomial of $J_{n}$. Nevertheless, their solution is important to solving spectral direct and especially inverse problems for the matrices of the form (2.9). Thus it makes sense to give some more detailed explanations of Theorem 2.2

Given a polynomial $p$ defined by (2.1) and satisfying the inequalities (2.6), the matrix $J_{n}$ such that $p(z)=\operatorname{det}\left(z E_{n}-J_{n}\right)$ can be reconstructed, for instance, by formulæ (2.8). However, one can also run a Sturm algorithm as it was noted in [16].

Indeed, let

$$
f_{0}(z):=p(z) \quad \text { and } \quad f_{1}(z):=\frac{q(z)}{b_{0}}
$$

where $q(z)$ is defined in (2.4). The polynomials $f_{0}$ and $f_{1}$ are monic, and $f_{1}$ is even or odd by construction. Now we construct a sequence of monic polynomials $f_{k}$, $\operatorname{deg} f_{k}=n-k$, by the following process

$$
\begin{aligned}
b_{1} f_{2}(z) & :=f_{0}(z)-\left(z+b_{0}\right) f_{1}(z), \\
b_{2} f_{3}(z) & :=f_{1}(z)-z f_{2}(z), \\
& \vdots \\
b_{n-2} f_{n-1}(z) & :=f_{n-3}(z)-z f_{n-2}(z), \\
b_{n-1} & :=f_{n-2}(z)-z f_{n-1}(z) .
\end{aligned}
$$

Thus, these equations give us all the entries $b_{k}$ of the matrix $J_{n}$ in (2.9). Moreover, the polynomials $f_{k}, k=1, \ldots, n$, are even or odd, and $f_{k}(z)$ is the characteristic polynomial of the principal submatrix of $J_{n}$ obtained by deleting first $k$ rows and columns.

We, finally, investigate the structure of the matrix (2.9) in detail. Again, let $p$ be its characteristic polynomial: $p(z)=\operatorname{det}\left(z E_{n}-J_{n}\right)$. Consider the following auxiliary 
matrix

$$
A_{n}=\left[\begin{array}{cccccc}
0 & 1 & 0 & \ldots & 0 & 0 \\
-b_{1} & 0 & 1 & \ldots & 0 & 0 \\
0 & -b_{2} & 0 & \ldots & 0 & 0 \\
\vdots & \vdots & \vdots & \ddots & \vdots & \vdots \\
0 & 0 & 0 & \ldots & 0 & 1 \\
0 & 0 & 0 & \ldots & -b_{n-1} & 0
\end{array}\right]
$$

and its submatrix

$$
A_{n-1}=\left[\begin{array}{cccccc}
0 & 1 & 0 & \ldots & 0 & 0 \\
-b_{2} & 0 & 1 & \ldots & 0 & 0 \\
0 & -b_{3} & 0 & \ldots & 0 & 0 \\
\vdots & \vdots & \vdots & \ddots & \vdots & \vdots \\
0 & 0 & 0 & \ldots & 0 & 1 \\
0 & 0 & 0 & \ldots & -b_{n-1} & 0
\end{array}\right]
$$

obtained from $A_{n}$ by deleting its first row and column. It is easy to see that

$$
p(z)=\operatorname{det}\left(z E_{n}-J_{n}\right)=\operatorname{det}\left(z E_{n}-A_{n}\right)+b_{0} \operatorname{det}\left(z E_{n-1}-A_{n-1}\right)
$$

It is also clear that if $p(z)=p_{0}\left(z^{2}\right)+z p_{1}\left(z^{2}\right)$, where $p_{0}(u)$ and $p_{1}(u)$ are the even and odd parts of $p$, respectively, then

for $n=2 l$,

$$
p_{0}\left(z^{2}\right)=\operatorname{det}\left(z E_{n}-A_{n}\right) \quad \text { and } \quad z p_{1}\left(z^{2}\right)=b_{0} \operatorname{det}\left(z E_{n-1}-A_{n-1}\right)
$$

for $n=2 l+1$,

$$
z p_{1}\left(z^{2}\right)=\operatorname{det}\left(z E_{n}-A_{n}\right) \quad \text { and } \quad p_{0}\left(z^{2}\right)=b_{0} \operatorname{det}\left(z E_{n-1}-A_{n-1}\right)
$$

These formulæ imply the following simple fact.

Proposition 2.3. Let the polynomial $p(z)=p_{0}\left(z^{2}\right)+z p_{1}\left(z^{2}\right)$ be the characteristic polynomial of the matrix $J_{n}$ given in (2.9), i.e. $p(z)=\operatorname{det}\left(z E_{n}-J_{n}\right)$. Then the polynomial $f(z)=(-1)^{\left[\frac{n+1}{2}\right]}\left[p_{0}\left(-z^{2}\right)+(-1)^{n} z p_{1}\left(-z^{2}\right)\right]$ is the characteristic polynomial of the matrix

$$
\left[\begin{array}{cccccc}
b_{0} & 1 & 0 & \ldots & 0 & 0 \\
b_{1} & 0 & 1 & \ldots & 0 & 0 \\
0 & b_{2} & 0 & \ldots & 0 & 0 \\
\vdots & \vdots & \vdots & \ddots & \vdots & \vdots \\
0 & 0 & 0 & \ldots & 0 & 1 \\
0 & 0 & 0 & \ldots & b_{n-1} & 0
\end{array}\right]
$$


Here $[\alpha]$ denotes the maximal integer not exceeding $\alpha$.

Proof. We prove the proposition for $n=2 l$. For $n=2 l+1$ it can be proved analogously.

Let $n=2 l$. Then $\left[\frac{n+1}{2}\right]=l$ and the polynomial $q$ has the form

$$
f(z)=(-1)^{l} p_{0}\left(-z^{2}\right)+(-1)^{l} z p_{1}\left(-z^{2}\right)
$$

Using formulæ (2.10) one can obtain

$$
(-1)^{l} p_{0}\left(-z^{2}\right)=(-1)^{l} \operatorname{det}\left(-i z E_{n}-A_{n}\right)=\operatorname{det}\left(z E_{n}-i A_{n}\right)=\operatorname{det}\left(z E_{n}-B_{n}\right),
$$

where

$$
B_{n}=\left[\begin{array}{cccccc}
0 & 1 & 0 & \ldots & 0 & 0 \\
b_{1} & 0 & 1 & \ldots & 0 & 0 \\
0 & b_{2} & 0 & \ldots & 0 & 0 \\
\vdots & \vdots & \vdots & \ddots & \vdots & \vdots \\
0 & 0 & 0 & \ldots & 0 & 1 \\
0 & 0 & 0 & \ldots & b_{n-1} & 0
\end{array}\right]
$$

Here we used the fact (see e.g. [10, Ch. II]) that the characteristic polynomial of any tridiagonal matrix does not depend on the $(i+1, i)$ th and $(i, i+1)$ th entries separately but on their product, so the matrices $i A_{n}$ and $B_{n}$ have the same characteristic polynomial.

Analogously we have

$$
\begin{gathered}
(-1)^{l} z p_{1}\left(-z^{2}\right)=i(-1)^{l} b_{0} \operatorname{det}\left(-i z E_{n-1}-A_{n-1}\right)= \\
-b_{0} \operatorname{det}\left(z E_{n-1}-i A_{n-1}\right)=-b_{0} \operatorname{det}\left(z E_{n-1}-B_{n-1}\right),
\end{gathered}
$$

where

$$
B_{n-1}=\left[\begin{array}{cccccc}
0 & 1 & 0 & \ldots & 0 & 0 \\
b_{2} & 0 & 1 & \ldots & 0 & 0 \\
0 & b_{3} & 0 & \ldots & 0 & 0 \\
\vdots & \vdots & \vdots & \ddots & \vdots & \vdots \\
0 & 0 & 0 & \ldots & 0 & 1 \\
0 & 0 & 0 & \ldots & b_{n-1} & 0
\end{array}\right]
$$

Thus, we get

$$
f(z)=\operatorname{det}\left(z E_{n}-B_{n}\right)-b_{0} \operatorname{det}\left(z E_{n-1}-B_{n-1}\right),
$$

so $f(z)$ is the characteristic polynomial of the matrix (2.11). $\mathrm{\square}$ 
3. Some solved direct and inverse spectral problems for the Schwarz matrices. In the previous section, we described properties of the characteristic polynomials of the Schwarz matrices of the form (2.9) and recall methods of reconstruction of such matrices from their characteristic polynomials. However, we are interested in direct and inverse spectral problems of the Schwarz matrices.

It is natural to study a dependence of the spectrum of the matrix $J_{n}$ given in (2.9) in terms of signs of the entries $b_{k}$ of this matrix. Since we have the relations (2.8)

$$
\begin{aligned}
& b_{0}=\Delta_{1}(p), \\
& b_{k}=\frac{\Delta_{k-2}(p) \Delta_{k+1}(p)}{\Delta_{k-1}(p) \Delta_{k}(p)}, \quad k=1, \ldots, n-1,
\end{aligned}
$$

between the entries $b_{k}$ of the matrix $J_{n}$ and the coefficients $a_{j}$ of its characteristic polynomials, it makes sense to use results of the theory of root location of polynomials that use signs of Hurwitz minors.

The most known such result is the famous Hurwitz theorem stating that a real polynomial $p(z)$ has all its zeroes in the open left half-plane of the complex plane if and only if its Hurwitz minors are positive (see (2.5)). We recall that a polynomial is called Hurwitz stable if all its zeroes lie in the open left half-plane.

Using Hurwitz theorem H. Wall established the following fact in [18, p.314] (see also [16]).

Theorem 3.1 (Wall). The Schwarz matrix $J_{n}$ given in (2.9) has all its eigenvalues in the open left half-plane if all the entries $b_{k}$ are positive. Conversely, given a sequence of complex numbers $\lambda_{1}, \ldots, \lambda_{n}$ with negative real parts, there exists a unique Schwarz matrix $J_{n}$ of the form (2.9) with $b_{k}>0, k=0,1, \ldots, n-1$, such that $\sigma\left(J_{n}\right)=\left\{\lambda_{1}, \ldots, \lambda_{n}\right\}$. Here $\sigma\left(J_{n}\right)$ denotes the spectrum of the matrix $J_{n}$.

Thus this theorem solves the direct and inverse problems for stable Schwarz matrices, that is, the Schwarz matrices with positive $b_{k}$. Since any stable matrix is similar to a stable Schwarz matrix [16, such Schwarz matrices are sometimes called Routh canonical forms (see e.g. [15, 5]).

The next result regarding eigenvalue location of the matrix (2.9) is based on the so-called Routh-Hurwitz theorem established by Gantmacher in 12, Theorem 4, p. 230].

Theorem 3.2 (Routh-Hurwitz). Let the polynomial $p$ be defined in (2.1) and satisfy (2.6). The number $m$ of roots of $p$ lying in the open right half-plane is given 
by the formula

$$
m=v\left(1, \Delta_{1}(p), \frac{\Delta_{2}(p)}{\Delta_{1}(p)}, \frac{\Delta_{3}(p)}{\Delta_{2}(p)}, \ldots, \frac{\Delta_{n}(p)}{\Delta_{n-1}(p)}\right)
$$

or equivalently

$$
m=v\left(1, \Delta_{1}(p), \Delta_{3}(p), \ldots\right)+v\left(1, \Delta_{2}(p), \Delta_{4}(p), \ldots\right),
$$

where $\Delta_{j}(p)$ are the Hurwitz determinants of $p$, and $v\left(c_{0}, c_{1}, \ldots, c_{l}\right)$ denotes the number of sign changes in the sequence $\left[c_{0}, c_{1}, \ldots, c_{l}\right]$.

Remark 3.3. Note that in Theorem 3.2, all Hurwitz determinants of the polynomial $p$ are nonzero by assumption, so we use the standard calculation of the sign changes in the sequences of the Hurwitz determinants. However, this theorem is also true in the case when some of Hurwitz determinants of $p$ equal zero [12, $\S 8$, p. 235] (see also [14]).

Using Theorem 3.2 and formulæ (2.8) one can easily obtain the following result due to Schwarz [16, Satz 5] which also follows from Theorem D and formulæ (2.1) of Wall's work [18.

THEOREM 3.4. Given a real tridiagonal matrix $J_{n}$ as in (2.9), the number of negative terms in the sequence

$$
b_{0}, b_{0} b_{1}, b_{0} b_{1} b_{2}, \ldots, b_{0} b_{1} \cdots b_{n-1}
$$

is equal to the number of eigenvalues of $J_{n}$ in the open right half-plane of the complex plane.

This theorem uses sign patterns of the entries of the matrix $J_{n}$ to localize the distribution of its eigenvalues. So this result can be identified as the solution of a direct spectral problem of the matrix $J_{n}$. The inverse spectral problem is somewhat trivial in light of Theorems 2.2 and 3.2 and formulæ (2.8).

TheOREM 3.5. Let $\lambda_{1}, \ldots, \lambda_{n}$ be a sequence of complex numbers with $m$ numbers in the open right half-plane and $n-m$ numbers in the open left half-plane such that the polynomial $p(z)=\prod_{i=1}^{n}\left(z-\lambda_{i}\right)$ satisfies the inequalities (2.6):

$$
\Delta_{j}(p) \neq 0, \quad j=1, \ldots, n .
$$

There exists a unique matrix $J_{n}$ of the form (2.9) such that the number of negative terms in the sequence (3.1) constructed with the entries of $J_{n}$ equals $m$ and $\sigma\left(J_{n}\right)=$ $\left\{\lambda_{1}, \ldots, \lambda_{n}\right\}$.

Note that in Theorem 3.1 we did not need to suppose that the polynomial $p(z)=$ $\prod_{i=1}^{n}\left(z-\lambda_{i}\right)$ satisfies (2.6) , because all Hurwitz stable polynomials automatically satisfy 
these inequalities by the Hurwitz theorem we mentioned above (see also Theorem 3.2 and remark after it).

Theorem 3.1 deals with the Schwarz matrices with positive $b_{k}$, so it is natural to study the Schwarz matrices (2.9) with all negative $b_{k}$. This problem was solved by O. Holtz in [13, Corollary 2], where she obtained the following result.

Theorem 3.6 (Holtz). Let the matrix $J_{n}$ be defined in (2.9) with $b_{k}<0, k=$ $0, \ldots, n-1$. Then its eigenvalues $\lambda_{i}$ are simple real and satisfy the inequalities

$$
\lambda_{1}>-\lambda_{2}>\lambda_{3}>\cdots>(-1)^{n-1} \lambda_{n}>0 .
$$

Conversely, for any sequence of real numbers $\lambda_{1}, \ldots, \lambda_{n}$ distributed as in (3.2), there exists a unique matrix $J_{n}$ of the form (2.9) with $b_{k}<0, k=0, \ldots, n-1$, such that $\sigma\left(J_{n}\right)=\left\{\lambda_{1}, \ldots, \lambda_{n}\right\}$.

REMARK 3.7. Note that there is a mistake in the proof of the main theorem, Theorem 1, in [13]. Namely, the inequality (11) of [13] does not turn to equality for $n>3$ and $\lambda_{1}=-\lambda_{2}$ unlike it is claimed in [13], so the proof of (11) is not satisfactory. However, it is not difficult to prove that inequality (11) holds for $n>3$ and $\lambda_{1}=-\lambda_{2}$, so the statement of the theorem is valid.

Theorem 3.6 was proved in [1] by a technique different from one used in [13]. However, it can be proved easily using properties of generalized Hurwitz polynomials [17] (see Remark 4.10). We just note that in Theorem 3.6, there is no requirement for the polynomial $p(z)=\prod_{i=1}^{n}\left(z-\lambda_{i}\right)$ to satisfy the inequalities (2.6). As we will show, the polynomials with the distribution of zeroes as in (3.2) automatically satisfy (2.6), since they are dual (in the sense of Theorem 4.7) to Hurwitz stable polynomials.

Finally, we should mention that in [1, there was an attempt to solve the direct problem for the matrix (2.9) with $b_{0}<0, b_{1}>0$ and $b_{k}<0$ for $k=2, \ldots, n-1$. However, their result is incorrect. In [2], the authors published a correct version of their Theorem 9 without proof. Here we completely solved the direct and inverse problems for such matrices (see Theorem 6.5).

4. Generalized Hurwitz polynomials, basic properties. In this section, we define (almost) generalized Hurwitz polynomials [17] and review their basic properties that will be helpful to study spectral problems of the Schwarz matrices.

Definition 4.1. A real polynomial $p$ of degree $n$ is called generalized Hurwitz polynomial of type $I$ of order $\varkappa$, where $1 \leqslant \varkappa \leqslant\left[\frac{n+1}{2}\right]$, if it has exactly $\varkappa$ zeroes in the closed right half-plane, all of which are nonnegative and simple:

$$
0 \leqslant \mu_{1}<\mu_{2}<\cdots<\mu_{\varkappa}
$$


such that $p\left(-\mu_{i}\right) \neq 0, i=1, \ldots, \varkappa$, and $p$ has an odd number of zeroes, counting multiplicities, on each interval $\left(-\mu_{\varkappa},-\mu_{\varkappa-1}\right), \ldots,\left(-\mu_{3},-\mu_{2}\right),\left(-\mu_{2},-\mu_{1}\right)$. Moreover, the number of zeroes of $p$ on the interval $\left(-\mu_{1}, 0\right)$ (if any) is even, counting multiplicities. The other real zeroes lie on the interval $\left(-\infty,-\mu_{\varkappa}\right)$ : an odd number of zeroes, counting multiplicities, when $n=2 l$, and an even number of zeroes, counting multiplicities, when $n=2 l+1$. All nonreal zeroes of $p$ (if any) are located in the open left half-plane of the complex plane.

Thus, the order $\varkappa$ of a generalized Hurwitz polynomial of type I indicates the number of its zeroes in the closed right half-plane. Moreover, the zeroes of a generalized Hurwitz polynomial in the closed right half-plane structure the distribution of its negative zeroes. So not every real polynomial with only real simple zeroes in the closed right half-plane is generalized Hurwitz. The generalized Hurwitz polynomials of type I of order 0 are obviously Hurwitz stable polynomials, since they have no zeroes in the closed right half-plane.

The generalized Hurwitz polynomials of type II is a generalization of real polynomials with zeroes in the open right half plane.

Definition 4.2. A real polynomial $p(z)$ is generalized Hurwitz of type II if and only if the polynomial $p(-z)$ is generalized Hurwitz of type I.

It is clear that all results obtained for the generalized Hurwitz polynomials of type I can be easily reformulated for the generalized Hurwitz polynomials of type II. Thus, we formulate all results in this section only for generalized Hurwitz polynomials of type I.

The main fact about generalized Hurwitz polynomials we use in this paper is the following theorem.

Theorem 4.3 (Generalized Hurwitz theorem). The polynomial p given in (2.1) is generalized Hurwitz if and only if

$$
\Delta_{n-1}(p)>0, \Delta_{n-3}(p)>0, \Delta_{n-5}(p)>0, \ldots
$$

The order $\varkappa$ of the polynomial $p$ equals

$$
\varkappa=\mathrm{V}^{\mathrm{F}}\left(\Delta_{n}(p), \Delta_{n-2}(p), \ldots, 1\right) \quad \text { if } \quad p(0) \neq 0,
$$

or

$$
\varkappa=\mathrm{V}^{\mathrm{F}}\left(\Delta_{n-2}(p), \Delta_{n-4}(p), \ldots, 1\right)+1 \quad \text { if } \quad p(0)=0,
$$

where $V^{F}\left(c_{1}, \ldots, c_{n}\right)$ denotes the number of sign changes in the sequence $\left\{c_{1}, \ldots, c_{n}\right\}$ calculated in accordance with the Frobenius rule of signs. 
Let us recall a rule that was introduced by Frobenius for calculating the number of sign changes in a sequence of Hankel minors, [9] (see also [11, Ch. X, §10] and [14, Ch. 2]).

THEOREM 4.4 (Frobenius rule of signs). Given a sequence of real numbers $\left\{c_{1}, \ldots, c_{n}\right\}$, where $c_{1} c_{n} \neq 0$, if, for some $i$ and $j(0 \leqslant i \leqslant j)$,

$$
c_{i} \neq 0, \quad c_{i+1}=c_{i+2}=\cdots=c_{i+j}=0, \quad c_{i+j+1} \neq 0
$$

then the number $V^{F}\left(c_{1}, \ldots, c_{n}\right)$ of Frobenius sign changes must be calculated by assigning signs as follows:

$$
\operatorname{sign} c_{i+\nu}=(-1)^{\frac{\nu(\nu-1)}{2}} \operatorname{sign} c_{i}, \quad \nu=1,2, \ldots, j .
$$

Note that the number of Frobenius sign changes can be changed by the standard number of sign changes $v$ if all the terms of the sequence $\left\{c_{0}, c_{1}, \ldots, c_{n}\right\}$ are nonzero. In the sequel, we consider only polynomials with nonzero Hurwitz determinants (except the proof of Theorem 6.5), so in the rest of the paper (except Section 6), we use the standard number of sign changes $v$ instead of the number of Frobenius sign changes $V^{F}$. The formula (4.3) will not be used in the next sections.

By (4.2)-(4.3), $\varkappa=0$ if and only if $\Delta_{n-2 k}(p)>0, k=0,1, \ldots,\left[\frac{n-1}{2}\right]$. As we mentioned above, the generalized Hurwitz polynomials with $\varkappa=0$ are Hurwitz stable polynomials. Thus, Theorem 4.3 implies that a real polynomial $p$ of degree $n$ is Hurwitz stable if and only if $\Delta_{j}(p)>0, j=1, \ldots, n$. This is exactly the Hurwitz stability criterion.

On the other hand, the formulæ (4.2)-(4.3) imply that $\varkappa=\left[\frac{n+1}{2}\right]$ with $p(0) \neq 0$ if and only if

$$
(-1)^{d} \Delta_{n}(p)>0,(-1)^{d-1} \Delta_{n-2}(p)>0, \ldots, \quad \text { where } \quad d=\left[\frac{n+1}{2}\right] .
$$

In this case, the generalized Hurwitz polynomial $p$ of type I has neither nonreal nor multiple zeroes, so its zeroes are real and simple. Moreover, they are distributed as follows:

$$
0<\lambda_{1}<-\lambda_{2}<\lambda_{3}<\ldots<(-1)^{n-1} \lambda_{n}
$$

Definition 4.5. A real polynomial whose zeroes are distributed as in (4.5) is called self-interlacing of type I. 
Analogously to the general case, we introduce the self-interlacing polynomials of type II.

Definition 4.6. A polynomial $p(z)$ is called self-interlacing of type II if $p(-z)$ is self-interlacing of type I, or equivalently if its zeroes are distributed as follows:

$$
0<-\lambda_{1}<\lambda_{2}<-\lambda_{3}<\ldots<(-1)^{n} \lambda_{n} .
$$

From Definitions 4.54 4.6 it is easy to see that a real polynomial $p(z)$ is selfinterlacing (of type I or II) if and only if it has only real and simple zeroes interlacing the zeroes of the polynomial $p(-z)$.

Returning to Theorem 3.6 one can see from (3.2) that the characteristic polynomials of the matrices (2.9) with all $b_{k}<0$ are self-interlacing polynomials: of type I for odd $n$, and of type II for even $n$.

In 17 the following important fact about the relation (indeed, duality) between Hurwitz stable and self-interlacing polynomials was established. We will use this fact later to reveal a relation between Theorems 3.1 and 3.6 .

THEOREM 4.7. A polynomial $p(z)=p_{0}\left(z^{2}\right)+z p_{1}\left(z^{2}\right)$ is self-interlacing of type $I$ if and only if the polynomial $q(z)=p_{0}\left(-z^{2}\right)-z p_{1}\left(-z^{2}\right)$ is Hurwitz stable, where $p_{0}(u)$ and $p_{1}(u)$ are the even and odd parts of $p$, respectively (see (2.2)-(2.3)).

Indeed, there can be established a more general fact.

Theorem 4.8 ([17]). A polynomial $p(z)=p_{0}\left(z^{2}\right)+z p_{1}\left(z^{2}\right), p(0) \neq 0$, is generalized Hurwitz of order $\varkappa$ of type $I$ (type II) if and only if the polynomial $f(z)=p_{0}\left(-z^{2}\right)-z p_{1}\left(-z^{2}\right)$ is generalized Hurwitz of order $\left[\frac{n+1}{2}\right]-\varkappa$ of type I (type II), where $n=\operatorname{deg} p$.

The validity of this theorem follows from the simple observation that the order of a generalized Hurwitz polynomial changes as the number of nonnegative zeroes of its even and odd parts changes. So if, say, $\varkappa$ is the number of nonnegative zeroes of the polynomial $p_{0}(u)$ whose degree is $\left[\frac{n+1}{2}\right]$ as in Theorem 4.8, then the polynomial $p_{0}(-u)$ has exactly $\left[\frac{n+1}{2}\right]-\varkappa$ nonnegative zeroes.

Remark 4.9. From Definition 4.2 and Theorem 4.8 it is clear that if a real polynomial $p(z)=p_{0}\left(z^{2}\right)+z p_{1}\left(z^{2}\right)$ is generalized Hurwitz of type I, then the polynomial $p_{0}\left(-z^{2}\right)+z p_{1}\left(-z^{2}\right)$ is generalized Hurwitz of type II.

Remark 4.10. Theorem 3.1, Proposition 2.3, Theorem4.7 and Remark4.9 imply Theorem 3.6 .

Finally, let us introduce the so-called almost generalized Hurwitz polynomials. 
Definition 4.11. A real polynomial $p(z)$ is called almost generalized Hurwitz of order $\varkappa$ of type $I$ (type II) if the polynomial zp $(z)$ is generalized Hurwitz of order $\varkappa+1$ of type $I$ (resp. type II).

REMARK 4.12. Note that any almost generalized Hurwitz polynomial of order 0 of type I is a Hurwitz stable polynomial, while any almost generalized Hurwitz polynomial of type I of degree $2 l$ and of order $l$ is a self-interlacing polynomial of type II. Also any almost generalized Hurwitz polynomial of type II of degree $2 l+1$ and of order $l$ is a self-interlacing polynomial of type I.

For almost generalized Hurwitz polynomials we have the following basic theorem analogous to Theorem 4.3 (see [17]).

THEOREM 4.13. The polynomial p given in (2.1) is almost generalized Hurwitz if and only if

$$
\Delta_{n}(p)>0, \Delta_{n-2}(p)>0, \Delta_{n-4}(p)>0, \ldots
$$

The order $\varkappa$ of the polynomial $p$ equals

$$
\varkappa=\mathrm{V}^{\mathrm{F}}\left(\Delta_{n-1}(p), \Delta_{n-3}(p), \ldots, 1\right) .
$$

where $V^{F}\left(c_{1}, \ldots, c_{n}\right)$ denotes the number of sign changes in the sequence $\left\{c_{1}, \ldots, c_{n}\right\}$ calculated in accordance with the Frobenius rule of signs.

Note that almost generalized Hurwitz polynomials do not vanish at zero, so their orders equal the number of positive simple zeroes. One can easily describe the distribution of zeroes of almost generalized Hurwitz polynomials from Definitions 4.1 and 4.11. Moreover, if a real polynomial is generalized Hurwitz and almost generalized Hurwitz simultaneously, then it is Hurwitz stable [17].

5. Direct and inverse spectral problems for some Schwarz matrices. Let us again consider the Schwarz matrix

$$
J_{n}=\left[\begin{array}{cccccc}
-b_{0} & 1 & 0 & \ldots & 0 & 0 \\
-b_{1} & 0 & 1 & \ldots & 0 & 0 \\
0 & -b_{2} & 0 & \ldots & 0 & 0 \\
\vdots & \vdots & \vdots & \ddots & \vdots & \vdots \\
0 & 0 & 0 & \ldots & 0 & 1 \\
0 & 0 & 0 & \ldots & -b_{n-1} & 0
\end{array}\right]
$$

with all $b_{k}$ nonzero, and denote by $p(z)$ its characteristic polynomial, that is, $p(z)=$ $\operatorname{det}\left(z E_{n}-J_{n}\right)$. From formulæ (2.8) it is easy to obtain the following

$$
b_{0}=\Delta_{1}(p), \quad b_{2 j-1} b_{2 j}=\frac{\Delta_{2 j-3}(p) \Delta_{2 j+1}(p)}{\Delta_{2 j-1}^{2}(p)}, \quad j=1, \ldots,\left[\frac{n-1}{2}\right],
$$


and

$$
b_{2 j} b_{2 j+1}=\frac{\Delta_{2 j-2}(p) \Delta_{2 j+2}(p)}{\Delta_{2 j}^{2}(p)}, \quad j=0,1, \ldots,\left[\frac{n-2}{2}\right],
$$

where $\Delta_{-2}(p) \equiv 1$, and $[\alpha]$ denotes the maximal integer not exceeding $\alpha$.

From the formulæ (5.2) - (5.3) and from Theorems 2.2 and 4.3 it is easy to obtain the following fact.

TheOREM 5.1. Let the matrix $J_{n}$ be given in (5.1), and $n=2 l$. The characteristic polynomial $p$ of the matrix $J_{n}$ is generalized Hurwitz of type $I$ if and only if

$$
b_{0}>0, b_{1} b_{2}>0, b_{3} b_{4}>0, \ldots, b_{n-3} b_{n-2}>0 .
$$

The order $\varkappa$ of the polynomial $p$ is equal to the number of negative terms in the sequence

$$
b_{0} b_{1}, b_{0} b_{1} b_{2} b_{3}, b_{0} b_{1} b_{2} b_{3} b_{4} b_{5}, \ldots, b_{0} b_{1} \cdots b_{n-1} .
$$

Conversely, let $\lambda_{1}, \ldots, \lambda_{n}$ be a sequence of complex numbers such that the polynomial $p(z)=\prod_{k=1}^{n}\left(z-\lambda_{k}\right)$ is generalized Hurwitz of type I of order $\varkappa$ and satisfies the inequalities:

$$
\Delta_{j}(p) \neq 0, \quad j=1, \ldots, n .
$$

Then there exists a unique Schwarz matrix $J_{n}$ of the form (5.1) with entries $b_{k}$ satisfying (5.4) such that the number of negative terms in the sequence (5.5) is equal to $\varkappa$, and $\sigma\left(J_{n}\right)=\left\{\lambda_{1}, \ldots, \lambda_{n}\right\}$.

Proof. Let $p$ be the characteristic polynomial of the matrix $J_{n}$. It satisfies (5.6) by Theorem 2.2. Moreover, according to Theorem 4.3, it is generalized Hurwitz of type I if and only if $\Delta_{2 i-1}(p)>0$ for $i=1, \ldots, l$. By (5.3) these inequalities are equivalent to (5.4). Furthermore, from (5.3) we have

$$
\prod_{k=0}^{2 i-1} b_{k}=\frac{\Delta_{2 i}(p)}{\Delta_{2 i-2}(p)}, \quad i=1, \ldots, l .
$$

By Theorem 4.3, the order of the generalized Hurwitz polynomial $p$ is equal to the number of sign changes in the sequence $\Delta_{2}(p), \Delta_{4}(p), \ldots, \Delta_{2 l}(p)$. But from (5.7) we obtain that each sign change in this sequence corresponds to a negative number in the sequence (5.5).

Conversely, if the complex numbers $\lambda_{1}, \ldots, \lambda_{n}$ are such that the polynomial $p(z)=\prod_{k=1}^{n}\left(z-\lambda_{k}\right)$ is generalized Hurwitz of type I of order $\varkappa$ satisfying the inequalities (5.6), then by Theorems 2.2 and 4.3 and by formulæ (5.3) $-(5.2)$, there exists a 
unique matrix $J_{n}$ of the form (2.9) satisfying the inequalities (5.4) and with $\varkappa$ negative numbers in the sequence (5.5) such that its characteristic polynomial is $p$.

Analogously, using formulæ (5.2)-(5.3) and Theorems 2.2, 4.3, and 4.13 one can easily establish the following theorems.

Theorem 5.2. Let the matrix $J_{n}$ be given in (5.1), and $n=2 l+1$. The characteristic polynomial $p$ of the matrix $J_{n}$ is generalized Hurwitz of type I if and only if

$$
b_{0} b_{1}>0, b_{2} b_{3}>0, b_{4} b_{5}>0, \ldots, b_{n-3} b_{n-2}>0 .
$$

The order $\varkappa$ of the polynomial $p$ is equal to the number of negative terms in the sequence

$$
b_{0}, b_{0} b_{1} b_{2}, b_{0} b_{1} b_{2} b_{3} b_{4}, \ldots, b_{0} b_{1} \cdots b_{n-1} \text {. }
$$

Conversely, let $\lambda_{1}, \ldots, \lambda_{n}$ be a sequence of complex numbers such that the polynomial $p(z)=\prod_{k=1}^{n}\left(z-\lambda_{k}\right)$ is generalized Hurwitz of type I of order $\varkappa$ and satisfies the inequalities (5.6). Then there exists a unique Schwarz matrix $J_{n}$ of the form (5.1) with entries $b_{k}$ satisfying (5.8) such that the number of negative terms in the sequence (5.9) is equal to $\varkappa$, and $\sigma\left(J_{n}\right)=\left\{\lambda_{1}, \ldots, \lambda_{n}\right\}$.

THeOREM 5.3. Let the matrix $J_{n}$ be given in (5.1), and $n=2 l$. The characteristic polynomial $p$ of the matrix $J_{n}$ is almost generalized Hurwitz of type I if and only if

$$
b_{0} b_{1}>0, b_{2} b_{3}>0, b_{4} b_{5}>0, \ldots, b_{n-2} b_{n-1}>0 .
$$

The order $\varkappa$ of the polynomial $p$ is equal to the number of negative terms in the sequence

$$
b_{0}, b_{0} b_{1} b_{2}, b_{0} b_{1} b_{2} b_{3} b_{4}, \ldots, b_{0} b_{1} \cdots b_{n-2} \text {. }
$$

Conversely, let $\lambda_{1}, \ldots, \lambda_{n}$ be a sequence of complex numbers such that the polynomial $p(z)=\prod_{k=1}^{n}\left(z-\lambda_{k}\right)$ is almost generalized Hurwitz of type I of order $\kappa$ and satisfies the inequalities (5.6). Then there exists a unique Schwarz matrix $J_{n}$ of the form (5.1) with entries $b_{k}$ satisfying (5.10) such that the number of negative terms in the sequence (5.11) is equal to $\varkappa$, and $\sigma\left(J_{n}\right)=\left\{\lambda_{1}, \ldots, \lambda_{n}\right\}$.

TheOREM 5.4. Let the matrix $J_{n}$ be given in (5.1), and $n=2 l+1$. The characteristic polynomial $p$ of the matrix $J_{n}$ is almost generalized Hurwitz of type I if and only if

$$
b_{0}>0, b_{1} b_{2}>0, b_{3} b_{4}>0, \ldots, b_{n-2} b_{n-1}>0 .
$$

The order $\varkappa$ of the polynomial $p$ is equal to the number of negative terms in the sequence

$$
b_{0} b_{1}, b_{0} b_{1} b_{2} b_{3}, b_{0} b_{1} b_{2} b_{3} b_{4} b_{5}, \ldots, b_{0} b_{1} \cdots b_{n-2} \text {. }
$$


Conversely, let $\lambda_{1}, \ldots, \lambda_{n}$ be a sequence of complex numbers such that the polynomial $p(z)=\prod_{k=1}^{n}\left(z-\lambda_{k}\right)$ is almost generalized Hurwitz of type I of order $\kappa$ and satisfies the inequalities (5.6). Then there exists a unique Schwarz matrix $J_{n}$ of the form (5.1) with entries $b_{k}$ satisfying (5.12) such that the number of negative terms in the sequence (5.13) is equal to $\varkappa$, and $\sigma\left(J_{n}\right)=\left\{\lambda_{1}, \ldots, \lambda_{n}\right\}$.

REMARK 5.5. It is also easy to prove and formulate an analogous theorems for (almost) generalized Hurwitz polynomials of type II. But it is not necessary, since if the characteristic polynomial of a matrix $J_{n}$ is (almost) generalized Hurwitz of type II, then the characteristic polynomial of a matrix $-J_{n}$ is (almost) generalized Hurwitz of type I. But changing the sign of the matrix will change, in fact, just the sign of the entry $b_{0}$, since the characteristic polynomial of tridiagonal matrices depends on the products of the $(i, i+1)$ th and $(i+1, i)$ th entries [10, Ch. 2]. So if we change their signs simultaneously, this does not change the characteristic polynomial. Thus, if we have a matrix $J_{n}$ of the form (5.1) such that $b_{0}<0$ and $b_{0} b_{1}<0$, we should consider the matrix $-J_{n}$ and apply one of Theorems 5.15 .4 (if any).

Thus, we solved the direct and inverse spectral problems for matrices (5.1) in the case when the characteristic polynomials of such matrices are (almost) generalized Hurwitz. So given a matrix $J_{n}$ as in (5.1), if its entries satisfy conditions of one of the theorems of the present section, then its characteristic polynomial is (almost) generalized Hurwitz of certain order, and we can describe the distribution of eigenvalues of this matrix. Conversely, for any (almost) generalized Hurwitz polynomial $p$ with nonzero Hurwitz determinants one can always construct a matrix of the form (5.1) whose characteristic polynomial is $p$.

6. Examples. In this section, we show how the results of the previous section can be used for certain sign patterns of the Schwarz matrix (5.1).

Consider the following matrix

$$
S_{n}=\left[\begin{array}{ccccccccccc}
-a & 1 & 0 & \ldots & 0 & 0 & 0 & 0 & \ldots & 0 & 0 \\
-c_{1} & 0 & 1 & \ldots & 0 & 0 & 0 & 0 & \ldots & 0 & 0 \\
0 & -c_{2} & 0 & \ldots & 0 & 0 & 0 & 0 & \ldots & 0 & 0 \\
\vdots & \vdots & \vdots & \ddots & \vdots & \vdots & \vdots & \vdots & \ddots & \vdots & \vdots \\
0 & 0 & 0 & \ldots & -c_{k} & 0 & 1 & 0 & \ldots & 0 & 0 \\
0 & 0 & 0 & \ldots & 0 & c_{k+1} & 0 & 1 & \ldots & 0 & 0 \\
\vdots & \vdots & \vdots & \ddots & \vdots & \vdots & \vdots & \vdots & \ddots & \vdots & \vdots \\
0 & 0 & 0 & \ldots & 0 & 0 & 0 & 0 & \ldots & 0 & 1 \\
0 & 0 & 0 & \ldots & 0 & 0 & 0 & 0 & \ldots & c_{n-1} & 0
\end{array}\right]
$$


where $a \in \mathbb{R} \backslash\{0\}$, and $c_{j}>0$ for $j=1, \ldots, n-1$.

TheOREM 6.1. Let $p(z)$ be the characteristic polynomial of the matrix $S_{n}$ :

$$
p(z)=\operatorname{det}\left(z E_{n}-S_{n}\right) .
$$

- If $n=2 l+1$ and $k=2 m+1$ or $n=2 l$ and $k=2 m$, then $p(z)$ is generalized Hurwitz of order $\varkappa=l-m$ of type I (type II) provided $a>0$ (resp. $a<0$ ).

- If $n=2 l+1$ and $k=2 m$ or $n=2 l$ and $k=2 m-1$, then $p(z)$ is almost generalized Hurwitz of order $\varkappa=l-m$ of type I (type II) provided $a>0$ (resp. $a<0)$.

Proof. Without loss of generality suppose that $a>0$ (see Remark 5.5). From the conditions of the theorem and from the formulæ (2.8), we obtain that the characteristic polynomial $p$ of the matrix $S_{n}$ satisfies the inequalities

$$
\Delta_{k+2+4 i}(p)<0, \quad i=0,1, \ldots,\left[\frac{n-k-2}{4}\right],
$$

while all other Hurwitz determinants of $p$ are positive. The statement of the theorem now follows from these inequalities, from Theorems 4.3 and 4.13 , and from Definition 4.2 .

A converse theorem can also be established provided the given polynomial satisfies the inequalities (6.2) while its other Hurwitz determinants are positive.

TheOREM 6.2. Let $\lambda_{1}, \ldots, \lambda_{n}$ be a sequence of complex numbers such that the polynomial $p(z)=\prod_{i=1}^{n}\left(z-\lambda_{i}\right)$ is a generalized Hurwitz polynomial of order $\varkappa$ of type I such that

$$
\Delta_{n-2 \varkappa+2+4 i}(p)<0, \quad i=0,1, \ldots,\left[\frac{\varkappa-1}{2}\right]
$$

and other $\Delta_{j}(p)$ are positive. Then there exists a unique Schwarz matrix $S_{n}$ of the form (6.1) with $a>0$ and $k=n-2 \varkappa$ such that $\sigma\left(S_{n}\right)=\left\{\lambda_{1}, \ldots, \lambda_{n}\right\}$.

Proof. Indeed, by the conditions of the theorem, all the Hurwitz determinants of the polynomial $p$ are nonzero, so according to Theorem 3.5. there exists a Schwarz matrix of the form (5.1) whose spectrum is $\left\{\lambda_{1}, \ldots, \lambda_{n}\right\}$. But from the inequalities (6.3) (see also (6.2) ) and from the positivity of all other Hurwitz determinants of $p$, it follows that the sign pattern of this matrix must be as in (6.1) with $k=n-2 \varkappa$. $\square$

Analogously, one can prove the following theorem.

THeOREM 6.3. Let $\lambda_{1}, \ldots, \lambda_{n}$ be a sequence of complex numbers such that the 
polynomial $p(z)=\prod_{i=1}^{n}\left(z-\lambda_{i}\right)$ is almost generalized Hurwitz of order $\varkappa$ of type $I$ and

$$
\Delta_{n-2 \varkappa+1+4 i}(p)<0, \quad i=0,1, \ldots,\left[\frac{\varkappa-1}{2}\right],
$$

while other $\Delta_{j}(p)$ are positive. Then there exists a unique Schwarz matrix $S_{n}$ of the form (6.1) with $a>0$ and $k=n-2 \varkappa-1$ such that $\sigma\left(S_{n}\right)=\left\{\lambda_{1}, \ldots, \lambda_{n}\right\}$.

Remark 6.4. Note that the results due to H. Wall and O. Holtz (Theorems 2.1 and (3.6) follow from Theorems 6.1, 6.2 and 6.3 for $k=0$ and for $k=n-1$, respectively (see Remark 4.12).

Finally, we show how to apply Theorem 6.1 to a more particular case. Consider the following matrix studied in [1]

$$
J_{n}=\left[\begin{array}{cccccc}
a & 1 & 0 & \ldots & 0 & 0 \\
-c_{1} & 0 & 1 & \ldots & 0 & 0 \\
0 & c_{2} & 0 & \ldots & 0 & 0 \\
\vdots & \vdots & \vdots & \ddots & \vdots & \vdots \\
0 & 0 & 0 & \ldots & 0 & 1 \\
0 & 0 & 0 & \ldots & c_{n-1} & 0
\end{array}\right], \quad a>0, c_{j}>0 .
$$

By Theorem 6.1, the characteristic polynomial of this matrix is (almost) generalized Hurwitz polynomial of order $\varkappa=\left[\frac{n-1}{2}\right]$ of type II. In [1], the problem was posed to find the condition on a sequence of complex number to be the spectrum of the matrix (6.4). In [1, there was also an attempt to solve the direct problem. However, the authors made a mistake, and their solution is incorrect. In [2], they published a correct version of their result, without proof. The following theorem solves the direct and inverse problems for matrices of the form (6.4).

TheOREm 6.5. The eigenvalues $\lambda_{j}$ of the matrix (6.4) are distributed in one of the following ways:

1) $-\lambda_{1}>\lambda_{2}>-\lambda_{3}>\cdots>(-1)^{n} \lambda_{n-2}>0, \lambda_{n-1}=\bar{\lambda}_{n} \in \mathbb{C}$, and $\operatorname{Re} \lambda_{n}>0$;

2) $\lambda_{1} \geqslant \lambda_{2}>-\lambda_{3}>\lambda_{4}>-\lambda_{5}>\cdots>(-1)^{n} \lambda_{n}>0$;

3) for some $k, k=1, \ldots,\left[\frac{n-3}{2}\right]$,

$-\lambda_{1}>\lambda_{2}>\cdots>-\lambda_{2 k-1}>\lambda_{2 k} \geqslant \lambda_{2 k+1} \geqslant \lambda_{2 k+2}>\lambda_{2 k+3}>\cdots>$ $(-1)^{n-1} \lambda_{n-1}>(-1)^{n} \lambda_{n}>0$

4) $-\lambda_{1}>\lambda_{2}>-\lambda_{3}>\cdots>(-1)^{n} \lambda_{n-2} \geqslant \lambda_{n-1} \geqslant \lambda_{n}>0$, and $(-1)^{n} \lambda_{n-2}>$ $\lambda_{n-1}>0$ if $n=2 l+1$.

Conversely, let $\lambda_{1}, \ldots, \lambda_{n}$ be a sequence of complex numbers satisfying one of the four conditions above, and $\sum_{i=1}^{n} \lambda_{i}>0$. Then there exists a unique matrix $J_{n}$ of the 
form (6.4) such that $\sigma\left(J_{n}\right)=\left\{\lambda_{1}, \ldots, \lambda_{n}\right\}$.

Proof. As we already mentioned, the characteristic polynomial $p$ of the matrix (6.4) is generalized Hurwitz of order $\varkappa=\left[\frac{n-1}{2}\right]$ of type II (if $n=2 l+1$ ) or almost generalized Hurwitz of order $\varkappa=\left[\frac{n-1}{2}\right]$ of type II (if $n=2 l$ ), by Theorem 6.1 According to Definitions 4.11, 4.1 and 4.2, the eigenvalues of the matrix $J_{n}$ are distributed in one of the four ways described in the statement of the theorem. Additionally, from the form of the matrix (6.4) it follows that $\sum_{i=1}^{n} \lambda_{i}=a>0$.

Conversely, let $\lambda_{1}, \ldots, \lambda_{n}$ be a sequence of complex numbers satisfying one of the four conditions above, and $\sum_{i=1}^{n} \lambda_{i}>0$. Then the polynomial $p(z)=\prod_{i=1}^{n}\left(z-\lambda_{i}\right)$ is generalized Hurwitz of order $\varkappa=\left[\frac{n-1}{2}\right]$ of type II (if $n=2 l+1$ ) or almost generalized Hurwitz of order $\varkappa=\left[\frac{n-1}{2}\right]$ of type II (if $n=2 l$ ) by Definitions 4.11, 4.1 and 4.2 It is left to prove that $p$ satisfies the inequalities (5.6).

Let $n=2 l+1$. Since $q(z):=p(-z)$ is generalized Hurwitz of type I of order $\varkappa=\left[\frac{n-1}{2}\right]=l$ by Definition 4.2 , we have

$$
\Delta_{2}(q)>0, \Delta_{4}(q)>0, \ldots, \Delta_{2 l}(q)>0 .
$$

and

$$
l-1=V^{F}\left(1, \Delta_{1}(q), \Delta_{3}(q), \ldots, \Delta_{2 l+1}(q)\right) .
$$

But $\Delta_{1}(q)=-\sum_{i=1}^{n}\left(-\lambda_{i}\right)>0$, so $V^{F}\left(1, \Delta_{1}(q)\right)=0$ and therefore we have

$$
l-1=V^{F}\left(\Delta_{1}(q), \Delta_{2}(q), \ldots, \Delta_{2 l+1}(q)\right) .
$$

Now the Frobenius rule of sign, Theorem 4.4 requires all the determinants $\Delta_{3}(q)$, $\Delta_{5}(q), \ldots, \Delta_{2 l+1}(q)$ to be nonzero and to satisfy the inequalities

$$
(-1)^{i-1} \Delta_{2 i-1}(q)>0, \quad i=1, \ldots, l+1 .
$$

Otherwise, $V^{F}\left(\Delta_{1}(q), \Delta_{2}(q), \ldots, \Delta_{2 l+1}(q)\right) \leqslant l-1$.

From the inequalities (6.5)-(6.6), Theorem 5.2, and the formulæ (2.8) we obtain that there exists a unique matrix of the form

$$
\left[\begin{array}{cccccc}
-a & 1 & 0 & \ldots & 0 & 0 \\
-c_{1} & 0 & 1 & \ldots & 0 & 0 \\
0 & c_{2} & 0 & \ldots & 0 & 0 \\
\vdots & \vdots & \vdots & \ddots & \vdots & \vdots \\
0 & 0 & 0 & \ldots & 0 & 1 \\
0 & 0 & 0 & \ldots & c_{n-1} & 0
\end{array}\right], \quad a>0, c_{j}>0 .
$$


whose characteristic polynomial is $q$. Now Remark [5.5 gives us the assertion of the theorem for $n=2 l+1$. The case $n=2 l$ can be established analogously.

Note that the additional condition $\sum_{i=1}^{n} \lambda_{i}>0$ is substantial for the solution of the inverse problem for the matrix (6.4). If this number is negative, then the matrix must have another sign pattern. But if this number is zero, the inverse problem has no solution for Schwarz matrices.

Note that using results of Section 5 one can find more examples of sign patterns of Schwarz matrices with (almost) generalized Hurwitz characteristic polynomials if it necessary. At least, given a Schwarz matrix, one can always say if its characteristic polynomial is (almost) generalized Hurwitz or not.

In the present work, we reminded the reader that the problem to solve spectral problems for Schwarz matrices using sign patterns is connected to the problem of determining the root location of a polynomial by the signs of its Hurwitz determinant. We used recent results on the last problem to get some new results on the former one. Thus the further study of connections between zero distribution of polynomials and the signs of their Hurwitz determinants can contribute to the study the spectra of Schwarz matrices with respect to their sign patterns.

Acknowledgment. The author thanks N. Bebiano and C. da Fonseca for helpful discussions, and the referees for useful comments and criticism.

\section{REFERENCES}

[1] N. Bebiano and J. da Providência. Inverse problems for pseudo-Jacobi matrices: existence and uniqueness results. Inverse Problems, 27(2):025005, 2011.

[2] N. Bebiano and J. da Providência. Corrigendum: Inverse problems for pseudo-Jacobi matrices: existence and uniqueness results. Inverse Problems, 28(2):06951, 2012.

[3] C. Chen and H. Chu. A matrix for evaluating Schwarz's form, IEEE Trans. Automatic Control, 11(2):303-305, 1966.

[4] B. Datta. A constructive method for finding the Schwarz form of a Hessenberg matrix, IEEE Trans. Automatic Control, 19(5):616-617, 1974.

[5] B. Datta. On the similarity between a matrix and its Routh canonical form, IEEE Trans. Automatic Control, 20(2):273-274, 1975.

[6] B. Datta. An inertia theorem for the Schwarz matrix, IEEE Trans. Automatic Control, 20(2):274, 1975.

[7] B. Datta. On the computation of Routh canonical form of a Hessenberg matrix, IEEE Trans. Automatic Control, 20(4):571-572, 1975.

[8] L. Elsner and D. Hershkowitz. On the spectra of close-to-Schwarz matrices, Linear Algebra Appl., 363:81-88, 2003.

[9] G. Frobenius. Über das Trägheitsgesetz der quadratischen Formen. Sitz.-Ber. Acad. Wiss. Phys.-Math. Klasse, Berlin, 241-256; 407-431, 1953. 
[10] Ф.Р. Гантмахер, М. Г. Крейн. Осцилляционные матрицы и ядра и малые колебания механических систем. (Russian), Москва-Ленинград, ГТТИ, 1941.

English transl.: Oscillation matrices and kernels and small vibrations of mechanical systems. AMS Chelsea Publishing, Providence, RI, revised edition, 2002. Translation based on the 1941 Russian original. Edited and with a preface by Alex Eremenko.

[11] F. R. Gantmacher. The theory of matrices. Vol. 1. Translated from the Russian by K. A. Hirsch. Reprint of the 1959 translation. AMS Chelsea Publishing, Providence, RI, 1998.

[12] F. R. Gantmacher. The theory of matrices. Vol. 2. Translated by K. A. Hirsch Chelsea Publishing Co., New York, 1959.

[13] O. Holtz. The inverse eigenvalue problem for symmetric anti-bidiagonal matrices, Linear Algebra Appl., 408:268-274, 2005.

[14] O. Holtz and M. Tyaglov. Structured matrices, continued fractions, and root localization of polynomials. arXiv: 0912.4703, to appear in SIAM Review.

[15] N. Puri and C. Weygandt. Second method of Liapunov and Routh's canonical form, J. Franklin Inst., 276(5):365-384, 1963.

[16] H. R. Schwarz. Ein Verfahren zur Stabilitätsfrage bei Matrizen-Eigenwertproblemen, Zeitschrift für angew. Math. Phys., 7(6):473-500, 1956.

[17] M. Tyaglov. Generalized Hurwitz polynomials. arXiv:1005.3032, to appear in Complex analysis and Operator Theory.

[18] H. S. Wall. Polynomials whose zeros have negative real parts, Amer. Math. Mothly, 52(6):308322, 1945.

[19] H. S. Wall. Analytic Theory of Continued Fractions. D. Van Nostrand Company, Inc., New York, 1948. 